\title{
Monitoring the activity of the Be star OT Geminorum ${ }^{\star}$
}

\author{
A. Arellano Ferro ${ }^{1, \star \star}$, J.P. Sareyan ${ }^{2}$, J.J. Avila ${ }^{1}$, F. González ${ }^{1}$, M. Dumont ${ }^{3}$, and GEOS ${ }^{3}$ \\ [CHC, MAA, EYR, VRR, FDZ, KCH, MIS, PAR, BSQ, PMP, SCP] \\ 1 Departamento de Astronomía, IFUG, Universidad de Guanajuato Apdo. Postal 144, Guanajuato, Gto. México 36000, México \\ 2 Observatoire de la Côte d'Azur, Dept. Fresnel, BP. 229, 06304, Nice Cedex 4, France \\ 33 Promenade Venezia, 78000, Versailles, France
}

Received November 21, 1996; accepted May 27, 1997

\begin{abstract}
Observations obtained in 1995-1996 of the Be star OT Geminorum are reported and show that in october 1995 the star reached a very active phase with large variations around a bright plateau, a phase of activity we have also distinguished in previous observations dating from 1960.

The time scales involved are discussed and suggest that the violent variations of the activity propagate very quickly on the whole surface of the star or on a huge portion of it. No likely pulsation periods were found.

The assumption that the detected activity is due to the effects of an hypothetical companion leads to conclude that such companion could not be detected by the present interferometric techniques.
\end{abstract}

Key words: stars: emission lines, Be; activity; individual (OT Gem)

\section{Introduction}

The star OT Geminorum (HR 2817, HD 58050, B2Ve, $V=6.41$ in the Bright Stars Catalogue) was first reported to be variable -between photographic magnitude 6.3 and 6.0- by Hoffmeister (1934). Very little and scarce photoelectric photometry has been carried upon the star, and it concluded to the star constancy (in January 1982, Bozic et al. 1982), or only to the existence of night to night variations (from January to March 1982, Poretti 1982). Thus the hours time scale variations claimed by Figer (1981a) were not confirmed. Two long time scale sets of observations are available in the literature: a photographic monitoring carried out between 1960 and 1980 at Sonneberg Observatory (Berthold 1983), and the visual observations obtained by the GEOS (Groupe Européen

\footnotetext{
* Partly based on observations obtained at the La Luz Observatory of the University of Guanajuato, México.

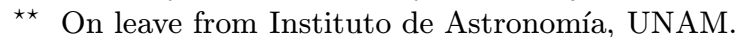

d'Observation Stellaire) during 1977 - 1980 and reported by Figer (1981b). Since that time, OT Gem has been followed by the GEOS as a part of a Be stars campaign, and professionals were alerted when they visually detected and confirmed the new light increase at JD 2449980-2450010 (i.e. in october 1995).

\section{The 1995 - 1996 observations}

\subsection{The La Luz data}

OT Gem has been observed from January to April 1996 with the $57 \mathrm{~cm}$ telescope at the La Luz Observatory (University of Guanajuato, Mexico, thereafter LLO). The technique used was differential photometry carried out with an Optec SSP-5 A photometer equipped with a Hamamatsu R1414 side-on photomultiplier and the standard $B$ and $V$ Johnson's filters. HR 2780 (HD 57049, $\mathrm{A} 2 \mathrm{Vn}, V=6.45)$ was the comparison star $\mathrm{C} 1$, and HR 2858 (HD 59059, B9IV, $V=6.22$ ) was the comparison star C2.

In order to show the night to night variations, the nightly measurements have been averaged. These averages and their standard deviation of the mean are plotted in Fig. 1. The top panel shows, in the intrumental system, the differences $\mathrm{OT}-\mathrm{C} 1$ in $V$ light, while the medium panel shows the same in $B-V$. The bottom panel displays the C1-C2 differences through the $V$ filter; all three panels are plotted at the same scale. The $\pm 1 \sigma$ error bars have an average size \pm 0.01 mag They are due to internal uncertainties that are driven mainly by improper centering or telescope track imperfections. Typically $20-40$ independent measurements were used to calculate the nightly means. The non-variable nature of both $\mathrm{C} 1$ and $\mathrm{C} 2$ is evident while well developed variations in OT Gem are observed.

The medium panel of Fig. 1 shows no variations in the color. This is of most importance since it implies no temperature variations in the stellar photosphere during the light variations. This strongly suggests that the light 
Table 1. Nightly mean values of the differences (OT Gem-C1) in Johnson's $V$-light

\begin{tabular}{ccc}
\hline JD $(2500000 .+)$ & $\begin{array}{c}\mathrm{OT}-\mathrm{C} 1(V) \\
(\mathrm{mag})\end{array}$ & $\begin{array}{c}\mathrm{rms} \\
(\mathrm{mag})\end{array}$ \\
\hline 93.736 & -0.307 & 0.009 \\
94.762 & -0.331 & 0.005 \\
96.768 & -0.303 & 0.008 \\
97.760 & -0.292 & 0.006 \\
100.709 & -0.304 & 0.011 \\
102.765 & -0.299 & 0.007 \\
103.755 & -0.326 & 0.010 \\
104.760 & -0.312 & 0.009 \\
105.783 & -0.307 & 0.011 \\
106.771 & -0.321 & 0.007 \\
107.763 & -0.334 & 0.011 \\
108.767 & -0.328 & 0.005 \\
109.715 & -0.348 & 0.002 \\
123.687 & -0.280 & 0.008 \\
132.719 & -0.262 & 0.010 \\
134.705 & -0.303 & 0.010 \\
136.732 & -0.253 & 0.007 \\
137.710 & -0.255 & 0.007 \\
139.702 & -0.212 & 0.009 \\
146.696 & -0.313 & 0.008 \\
147.697 & -0.337 & 0.009 \\
148.682 & -0.354 & 0.008 \\
149.691 & -0.366 & 0.006 \\
150.719 & -0.354 & 0.017 \\
153.676 & -0.369 & 0.007 \\
156.669 & -0.359 & 0.008 \\
157.675 & -0.404 & 0.011 \\
158.660 & -0.397 & 0.009 \\
159.668 & -0.395 & 0.009 \\
162.665 & -0.360 & 0.008 \\
167.663 & -0.339 & 0.011 \\
172.675 & -0.340 & 0.007 \\
173.651 & -0.337 & 0.011 \\
175.660 & -0.337 & 0.010 \\
176.633 & -0.289 & 0.008 \\
186.627 & -0.272 & 0.012 \\
187.607 & -0.288 & 0.006 \\
195.605 & -0.227 & 0.010 \\
\hline & &
\end{tabular}

variations are not due to classical radial or low order nonradial pulsations.

The night-to-night observations were explored in search of short-term variations. No variations above our internal accuracy of $\pm 0.01 \mathrm{mag}$ were observed in any of the nights.

High order $g$-modes could account for periods in the range of days (Dziembowski 1994) that we do not find here. An alternative scenario is of course that of stellar "spots" irregularly formed in time scales of the order of the light variations.
Poretti (1982) and Bozic et al. (1982), reported that no significant light or color variations during one night were observed. Likewise, we do not observe any significant period within the $2-5$ hours range, which was the typical length of the nights. Even after subtracting the mean of each night to each night data, no positive detection of a period was accomplished. The period searches were done using both Fourier analysis and the PDM method (Stellingwerf 1978).

In order to look for eventual periodicities in the night to night variations, we have performed a period analysis on the OT Gem-C1 differences in $V$ light data in the following manner: first the full set of individual observations were included and two frequencies were isolated: 0.012 cycles/day (period $P=83$ days) and $0.022 \mathrm{c} / \mathrm{d}(P=$ $45 \mathrm{~d}$ ). These can be interpreted as characteristic times of the long-term variation, and not as strict periodicities. Secondly, the same analysis was carried out on the nightly means of the top panel of Fig. 1. Essentially the same frequencies were found: $0.013 \mathrm{c} / \mathrm{d}(P=77 \mathrm{~d})$ and $0.021 \mathrm{c} / \mathrm{d}(P=48 \mathrm{~d})$.

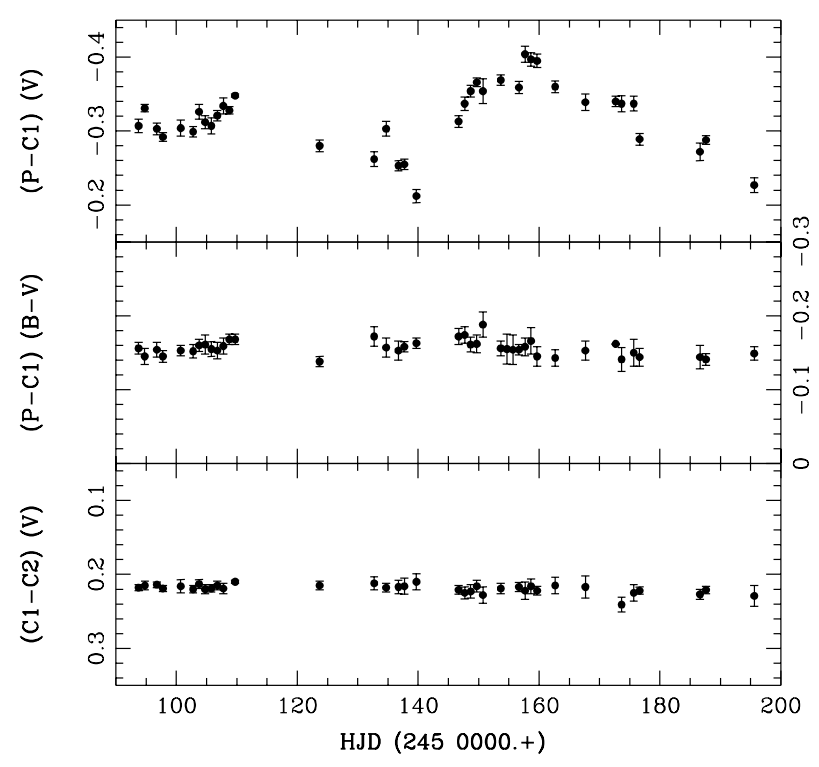

Fig. 1. 1996 observations of OT Gem carried out at La Luz Observatory. The top panel displays OT Gem (P)-HR 2780 (C1) light variations in Johnson's $V$ filter. The medium panel shows the same in $B-V$. The bottom panel shows HR 2780 (C1)-HR 2858 (C2) in $V$ filter

\subsection{The GEOS data}

While photometry was carried out in Mexico at LLO (38 nights, from JD 2450093 to 2450195), the GEOS continued observing from Europe, twelve observers providing 290 magnitude estimates obtained during more than 250 days (JD 2449971 to 2450226 , i.e. from september 11 th 
1995 to may 22nd 1996). As it is usually done with such observations, the original data for each observer has been corrected to a common zero, in order to account for the differences in chromatic sensitivity of each observer, related to the spectral type differences between comparison stars. The GEOS experience is that with such a procedure, errors in one observers' data reaching 0.2 on the visual magnitude are quite rare. In the case of OT Gem, and averaging severals observers' data as we did, the internal precision on the resulting measurement is about $0.1 \mathrm{mag}$, partly due to the differences in spectral type of the close comparisons that one has to use with the Argelander's method. This precision is of course inferior to that obtained by photoelectric photometry, but the scatter of the data can be further reduced by averaging the available measurements over 5 days (with the exception of the data where significant "events" appear). This seemed to us a reasonable compromise between the data precision and time resolution. These averaged data are reported in Table 2 .

The resulting GEOS light curve (Fig. 2) shows an internal dispersion lower than 0.05 magnitude, and it coincides quite well with the La Luz Observatory measurements, during the common observations of the winter (1996). This good correlation confirms that the GEOS data can be completely trusted, and particularly the observed light extrema.

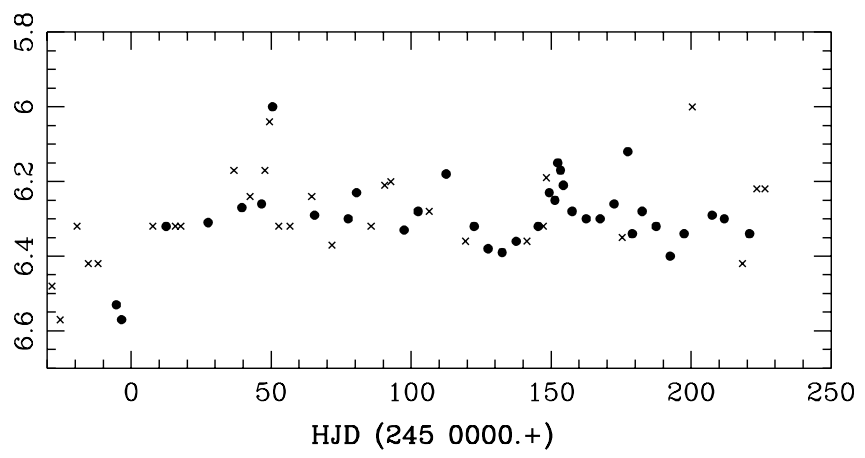

Fig. 2. 1995-96 GEOS visual observations of OT Gem. Crosses are single observations while the solid circles are averages of nearby multiple observations as described in the text

A mean behaviour can be clearly detected in this GEOS data and can be described as follows:

During the last 210 days of the GEOS observations, the star had a mean magnitude of $6.28 \pm 0.03$. However its regular mean magnitude has been for many years around $6.45 \pm 0.05$ (Dumont 1996), and it was this unexpected increase which called our attention to the star.

Fluctuations of about 0.1 magnitude do appear around this mean brightness:

Two well-marked minima are distinguished, i.e. a "wide" minimum at JD 2450130, and a sharper one at 2450192 , i.e. at a 60 days distance, with a duration of 25 and 15-20 days respectively.

There are two clear maxima, at JD $\sim 2450050$ and 2450152 , and possibly a third one at JD $\sim 2450112$ suggested by both sets of data but unfortunately not fully covered by the photoelectric data, i.e. these maxima are separated by 60 and 40 day intervals. The first maximum reaches a magnitude of 6.0 , while the second and third reach 6.2 and 6.15 respectively. The above intervals between maxima are not in contradiction with the times suggested by the analysis of the La Luz data in Sect. 2, specially considering the rather short time span of this data set (102 days).

\subsection{Light curve slopes}

To help establishing the activity level of the star, rough estimates of the slopes of the light increases and decreases can be given.

The rising branches observed in the light curve have a mean slope of $-13 \pm 3 \mathrm{mmag} /$ day. The simultaneous LLO and GEOS observations (JD 2450140-150) show the same $-11 \mathrm{mmag} /$ day, another confirmation that one can rely on the visual data.

The very unusual light increase at JD 2450048 has a slope of about $-70 \mathrm{mmag} /$ day and a steep decrease of about $+150 \mathrm{mmag} /$ day. This seems to be one of the most spectacular flare-like events in the star.

The light curve descending branches have a mean slope of $+13 \pm 3 \mathrm{mmag} /$ day.

\section{Comparison with previous long-time scale observations}

\subsection{The GEOS 1977-81 observations}

The GEOS 1977 to 1981 observations published by Figer (1981b) show that the star had remained at a visual magnitude $m_{v}$ around 6.4 for three years (JD 2443500 to 2444700). Then a 0.4 magnitude light increase (from $m_{v}=6.4$ to 6.0 ) was detected in november 1980 , followed by a rather rapid return to the previous light level, in the first months of 1981. The total event lasted 150-200 days. The $45 \%$ light increase has been correlated with simultaneous spectroscopy showing the Balmer discontinuity in emission (Figer 1981b), which indicates a very violent atmospheric event. The corresponding light curve slopes are about $-4 \mathrm{mmag} /$ day for the light increase (lasting about 60 days), and $+2.5 \mathrm{mmag} /$ day for the decrease (lasting about 90 days), i.e. much slower than those observed in 1996 and described in the previous section.

\subsection{The Sonneberg Observatory / Berthold observations}

A photographic monitoring of OT Gem has been carried out during 20 years at Sonneberg Observatory and published by Berthold (1983): it shows "slow irregular waves, 
Table 2. GEOS 1995-96 observations

\begin{tabular}{ccc|ccc|ccc}
\hline JD $(2499000 .+)$ & $m_{v}$ & $N$ & JD $(2499000 .+)$ & $m_{v}$ & $N$ & JD $(2499000 .+)$ & $m_{v}$ & $N$ \\
\hline 971.644 & 6.48 & 1 & 1065.500 & 6.29 & 2 & 1152.385 & 6.15 & 3 \\
974.649 & 6.57 & 1 & 1071.685 & 6.37 & 1 & 1153.352 & 6.17 & 5 \\
980.654 & 6.32 & 1 & 1077.500 & 6.30 & 10 & 1154.339 & 6.21 & 3 \\
984.663 & 6.42 & 1 & 1080.488 & 6.23 & 3 & 1157.500 & 6.28 & 8 \\
988.162 & 6.42 & 2 & 1085.702 & 6.32 & 1 & 1162.500 & 6.30 & 11 \\
994.682 & 6.53 & 6 & 1090.497 & 6.21 & 3 & 1167.500 & 6.30 & 8 \\
996.553 & 6.57 & 9 & 1092.798 & 6.20 & 2 & 1172.500 & 6.26 & 9 \\
1007.682 & 6.32 & 1 & 1097.500 & 6.33 & 20 & 1175.365 & 6.35 & 2 \\
1012.500 & 6.32 & 5 & 1102.500 & 6.28 & 14 & 1177.370 & 6.12 & 4 \\
1015.694 & 6.32 & 1 & 1106.416 & 6.28 & 1 & 1178.985 & 6.34 & 3 \\
1017.696 & 6.32 & 1 & 1112.500 & 6.18 & 9 & 1182.500 & 6.28 & 12 \\
1027.500 & 6.31 & 5 & 1119.429 & 6.36 & 1 & 1187.500 & 6.32 & 11 \\
1036.708 & 6.17 & 1 & 1122.500 & 6.32 & 20 & 1192.500 & 6.40 & 9 \\
1039.536 & 6.27 & 6 & 1127.500 & 6.38 & 5 & 1197.500 & 6.34 & 3 \\
1042.500 & 6.24 & 3 & 1132.500 & 6.39 & 8 & 1200.349 & 6.00 & 1 \\
1046.591 & 6.26 & 4 & 1137.500 & 6.36 & 9 & 1207.500 & 6.29 & 4 \\
1047.712 & 6.17 & 1 & 1141.438 & 6.36 & 1 & 1211.845 & 6.30 & 2 \\
1049.474 & 6.04 & 2 & 1145.364 & 6.32 & 7 & 1218.330 & 6.42 & 1 \\
1050.485 & 6.00 & 4 & 1147.272 & 6.32 & 1 & 1220.844 & 6.34 & 2 \\
1052.713 & 6.32 & 1 & 1148.274 & 6.19 & 1 & 1223.363 & 6.22 & 1 \\
1056.703 & 6.32 & 1 & 1149.310 & 6.23 & 5 & 1226.362 & 6.22 & 1 \\
1064.480 & 6.24 & 2 & 1151.342 & 6.25 & 4 & & & \\
\hline
\end{tabular}

Notes: $N$ is the number of individual observations included in the average.

superimposed by shorter variations". Given that this very extensive work was published in a rather difficult to find journal, we will briefly describe here the observed light behaviour.

During the years 1960 - 1980, the star had a mean photographic magnitude $m_{\mathrm{pg}}$ of $6.10 \pm 0.02$, for uninterrupted periods lasting between 300 and 800 days. This represents about $25 \%$ of the total observed time, and we will call it unexcited state.

Very often, however, the star showed light increases to $m_{\mathrm{pg}}=5.92 \pm 0.02$ (seven times in 20 years, or $12 \%$ of the time). In two occasions these light increases were followed by a "plateau", that lasted for about 300 days. Two more increases reached $m_{\mathrm{pg}}=5.86 \pm 0.02$. They were followed by a slow -i.e. lasting around 1000 days- light decrease to the mean 6.10 value of $m_{\mathrm{pg}}$. Once even the magnitude 5.7 was reached, this last value coinciding with the GEOS observations on winter 1980-81.

During these same 20 years, there were two light decreases below the 6.10 mag level, to magnitude $m_{\mathrm{pg}} \sim$ $6.2-6.25$. They lasted about 130 days between the beginning and the end of the decrease.

So, although the light variations seem to occur randomly, a general behaviour above or under the mean $m_{\mathrm{pg}}$ magnitude of 6.10 can be summarized as follows:
Frequent $20 \%$ light increases that last from 40 to 170 days in total. The light curve can also reach a plateau that lasts about 300 days.

When a more violent -i.e. a 25\%- light increase occurs, it is generally followed by a slow $\sim 1000$ days decrease.

The rare light minima - i.e. a light decreases of 20 to $25 \%$ - do not last more than 130 days. They only represent about $3 \%$ in the total time span of the observations, and do not seem to follow the light maxima.

We tried to define the time scales involved in these variations, and found the following:

The light increase rate is $-4.3 \pm 1.8 \mathrm{mmag} /$ day on the average. For the light increase observed at the end of 1980 both at Sonneberg and by the GEOS, we again get very similar values, respectively -3.2 and $-4 \mathrm{mmag} /$ day, even with a very small number of available data.

The light decrease rate is generally $+2.0 \pm 0.7$ $\mathrm{mmag} /$ day over a few weeks. It can also be the slow rate already mentioned: $+0.26 \pm 0.06 \mathrm{mmag} /$ day over $\sim 1000$ days. These rates are much slower than those we observed in 1995-96 (Table 3).

A remark should be made on the figures of Table 3. On the data of the previous observations, the time resolution is not as good as for those of $1995-96$, so the rates of variation are very probably somewhat underestimated, but the qualitative difference between excited and unexcited state remains. 
Table 3. Rates of the light variations in OT Gem

\begin{tabular}{lll}
\hline Departure from: & $\begin{array}{l}\text { Light increases } \\
\text { (in mmag/day) }\end{array}$ & $\begin{array}{l}\text { Light decreases } \\
\text { (in mmag/day) }\end{array}$ \\
\hline
\end{tabular}

\begin{tabular}{l} 
Unexcited state \\
$\begin{array}{l}\text { (during the years } \\
1960-80 \text { and 1980-81) }\end{array}$ \\
$\begin{array}{l}\text { Excited state } \\
\text { (plateau, }\end{array}$ \\
\begin{tabular}{ll} 
years 1995-96) & $\sim-4$ \\
\hline
\end{tabular} \\
\hline
\end{tabular}

\section{Discussion}

Given the fact that the 1995-96 mean magnitude is $\sim 0.17$ brighter than the "normal" magnitude 6.45 observed for many years, we suggest that these observations were obtained on a plateau similar to those observed by Berthold, that were also 0.18 magnitude above the "normal" $m_{\mathrm{pg}}=$ 6.10 , and that we have already described.

The latest plateau began in October 1995, and continued at least until May 1996, i.e. for 250 days.

We shall call here "active" the lengths of time during which the star undergoes short time scale light variations, i.e. the phases where its luminosity is above its "normal" level at $m_{v}=6.45 \mathrm{mag}$.

This activity is usually more violent when the star is already in an excited state, i.e. during the plateau phases. During these periods, the energy output of the star in the visible part of the spectrum can peak at $30 \%$ above the plateau value, which is already $\sim 18 \%$ higher than the unexcited state.

The increase and decrease rates of the variations in 1995-96 are about equal, about 3 times (light increases) and 6 times (light decreases) faster than away from the plateaus (see Table 1 ).

One should note that the plateaus observed since 1960 are separated by 5000 to 7000 days, i.e. about 14 to 19 years, a value quite different from the previous mentioned time scale for the variations of the spectroscopic emission (about 35 years according to Delplace \& Hubert 1975).

In its unexcited state, OT Gem shows light increases about twice as fast as the following decreases (with the exception of the very long "slow" $1000 \mathrm{~d}$. decreases).

Thus the variations observed during this 1995 - 1996 campaign are indeed very likely to be fluctuations around a plateau value.

One can wonder about an eventual correlation between the activity defined in the present paper on the basis of short time scale light variations - the star being significantly brighter than in its 'normal' unexcited state -, and the classical spectroscopic activity as measured by the intensity of emission in the lines of the Balmer series. Unfortunately the published spectroscopic data on
OT Gem are even more scarce than the photometric data. On the one hand, only a few measurements have been published: e.g. $\mathrm{H} \alpha$ intensities of 4.3 on October 22, 1981 (Andrillat 1983) and 3.2 on February 6, 1986 (Doazan et al. 1991) or $\mathrm{H} \beta$ profiles in 1966-67 (Kogure 1969). On the other hand, the "Un Atlas des étoiles Be" (Hubert \& Hubert 1979) gives a summary of the monitoring, carried out from 1954 to 1975, of the level of the spectroscopic activity of OT Gem.

We have not found any correlation between the light variations measured by Berthold (1983) and the spectroscopic activity as described in the Atlas. However the indications in the Atlas are qualitative estimates, at best on a year-to-year basis, without precise dates, which is obviously not suited to the present study of the short-term variations. Thus, the only clearly confirmed correlation between an increase of the spectroscopic activity and an increase of the light output is the one mentioned by Figer (1981b) and already described in Sect. 3.

Some more information about the nature of the variations can be derived from the probable physical parameters of the star:

OT Gem being a B2V star, its mass and radius can be respectively estimated as $10.3 \pm 3.5 M_{\odot}$ and $6.0 \pm 0.2 R_{\odot}$ (Underhill \& Doazan 1982), the gravity as log $g=3.85-$ 4.0 (Underhill \& Doazan 1982; Singh \& Chaubey 1987), and the effective temperature as $22500 \mathrm{~K}$ (Tur et al. 1995). The $v \sin i$ is $130 \mathrm{~km} / \mathrm{s}$ (Briot 1986; Slettebak 1994). The evaluation of $i$ by a method based on a comparison of the equivalent widths of visible and UV lines (Ruusalepp 1989) gives $i=60^{\circ}$ to $80^{\circ}$. This range of $i$ values is confirmed by the absence of shell lines (Hanuschik 1996). So we can expect the equatorial velocity to be around $140 \mathrm{~km} / \mathrm{s}$, which gives a probable rotation period of $2.2 \pm 0.2$ days.

Thus OT Gem is not among the fastests Be rotators, and apparently its light is not modulated by the rotation, as we do not detect any single or multiple wave light curve periodicity in the range of the possible rotation period.

Given the rather short time involved in the 1995-96 light increases and decreases, and the apparent absence of rotational modulation, the recurent 60 (or 40) days distance between light maxima or minima shows that the light variations must concern the whole, or a very large part, of the star's photosphere, i.e. the activity seems to grow in a time scale of a few rotations. The same is true, in general, for the damping of such activity.

In the particularly excited state of our 1995-96 observations, a large quantity of energy is involved in very short times, as the energy output increases are limited to a few days for the most violent events.

Poretti (1982) classified the star as $\gamma$ Cas type: however, in $\gamma$ Cas (B0 IVe) the light variations are less frequent (occuring typically over decades) and of a much larger amplitude (i.e. more than one magnitude, see Doazan et al. 1983) than those observed in Figs. 1 and 2. Furthermore 
the intensity of the emission at $\mathrm{H} \alpha$ reaches 5 in $\gamma$ Cas while in most cases it reaches 3 in OT Gem. Thus OT Gem (B2 Ve) might be considered as a "mild- $\gamma$ Cas".

What could be the short time scale limit of the activity in this star? We have seen that the most prominent maxima are attained in a few days (light curve slopes $\sim 70-150 \mathrm{mmag} /$ day or more). However there is no present evidence of any shorter time scale event. Spectroscopic observations at the time of these prominent maxima would be of course of paramount importance, as we know that in some Be stars very violent events can take place during a few minutes, like the $\mathrm{H} \alpha$ emission peak observed by Gosh et al. (1986) in o Andromedae, which was interpreted as mass being ejected.

As we have no "internal structure" explanation or modelization of the activity in OT Gem, the idea that the activity could be triggered by tidal effects due to the approaching of an unseen companion on an eccentric orbit is attractive and should be explored. Of course the period should be consistent with the time scales of 40 or 80 days, or even the 5000 to 7000 days between plateaus. If OT Gem "without known companion" (Abt \& Cardona 1984 ) is indeed a binary system, a secondary main sequence companion in the 1 to $6 M_{\odot}$ range would appear 7 to 2 magnitudes fainter than the $10 M_{\odot}$ primary. With an absolute magnitude $M_{v}=-2.5$ (Underhill \& Doazan 1982), the distance to OT Gem is $\sim 600$ parsecs. With a period of about 60 days or in the 5000 to 7000 days ranges the separation should be about 1 milliarcseconds (semi major axis $a / 2 \sim 0.6 \mathrm{AU})$ or 23 milliarcseconds $(a / 2 \sim$ $14 \mathrm{AU})$. These separations are below, or at the very limit of the present speckle or several telescopes interferometry precision thresholds.

Spectroscopy could bring an answer if superimposed on the bottom of broad photospheric lines of the primary, we could detect the signature of an eventual slow rotating A - F type companion.

\section{Conclusions}

The light variations of the star show, over the last 35 years, a few brief light minima, and frequent light maxima sometimes followed by a long plateau. The three observed plateaus are separated by $\sim 14$ and $\sim 19$ years. These plateaus represent the longest time spans of permanent strong activity in the star.

Our 1996 observations seem to be situated on a plateau. We do not detect any true long time scale periodicity, but rather time constants of the order of 40 to 80 days. Although sometimes violent, the light fluctuations have been limited to variations of 20 to $30 \%$ above and under the plateau level.

The fact that we do not detect here periods of the order of the rotational period ( $\sim 2$ days) means that the activity is not regionally localized on the stellar surface but rather longitudinally extended around the star, and that it develops very quickly.

Of course such behavior needs a careful simultaneous spectroscopic monitoring, in order to correlate the classical Be emission activity with the present knowledge of the light variations. These observations should be made especially at the phases where the activity increases rapidly, and at the light minima.

The assumption that the periodicities of about 60 days or even the time between plateaus, 5000 and 7000 days, are due to the modulation of the activity by an unseen companion, leads to conclude that this hypothetical companion cannot be detected with the present interferometric techniques. Only devoted spectroscopy could allow the detection of a companion.

Note added in proof: Observations carried out since september 1996 show that OT Gem has reached again its "normal" state luminosity (i.e. around magnitude 6.45). Thus, the 1995-96 plateau has had the same $\sim 300$ days duration mentioned above for other observed plateaus (between 250 and 340 days). Therefore 300 days seems to be a significant time constant of the plateau phases of the star activity.

Acknowledgements. The authors acknowledge C. Bourgois, J. Chauville, S. González-Bedolla, J.H. Peña and D. Briot for their help and/or hospitality through the different stages of this investigation. We thank the anonymous referee for his/her help and suggestions to improve the original manuscript. This work was partially supported by CONACYT (Mexico) projects 1219E9203, 458100-5-0464PE and F689E9411 and by the CNRS (France).

\section{References}

Abt H.A., Cardona O., 1984, ApJ 285, 190

Andrillat Y., 1983, A\&A 53, 319

Berthold Th., 1983, Mitt. Veranderliche Sterne 9 (Heft 4), 127

Bozic H., Muminovic M., Pavlovski K., Stupar M., Harmanec P., Horn J., Koubsky P., 1982, IBVS No. 2123

Briot D., 1986, A\&A 163, 67

Delplace A.M., Hubert H., 1975, A\&A 38, 75

Doazan V., Franco M., Rusconi L., Sedmak G., Stalio R., 1983, A\&A 128,171

Doazan V., Sedmak, G., Barylak M., Rusconi, L. 1991, "A Be star Atlas", ESA SP-1147

Dumont M., 1996 (private communication)

Dziembowski W.A., 1994, in Pulsation,mass loss and rotation in the Early Type Stars, Balona L.A., Henrichs H.F. and Le Contel J.M., (eds.) IAU Symp. No. 162, p. 55

Figer A., 1981a, GEOS Circular SA1

Figer A., 1981b, Workshop on Pulsating B stars, Nice, p. 237

Gosh K.K., Jaykumar K., Rosario M.J., Appakuty M., 1986, IBVS, No. 2897

Hanuschik R.W., 1996, A\&A 308, 170 
Hoffmeister C., 1934, AN 253, 195

Hubert A.M., Hubert H., 1979, "Un Atlas des étoiles Be", Meudon Observatory

Kogure T., 1969, A\&A 1, 253

Poretti E., 1982, IBVS, No. 2129

Ruusalepp M., 1989, Tartu Astrophys. Obs. Teated 100, 1
Singh M., Chaubey U.S., 1987, Astrophys. Space Sci. 129, 251

Slettebak A., 1994, ApJ 94, 163

Stellingwerf R., 1978, ApJ 224, 953

Tur N.S., Goraya P.S., Sharma S.D., 1995, PASP 107, 730

Underhill A.B., Doazan V., 1982, in: B Stars with and without emission lines (NASA SP456) 